\title{
Image-guided intensity-modulated radiotherapy for patients with locally advanced gastric cancer: a clinical feasibility study
}

\author{
Harun Badakhshi • Arne Gruen • Reinhold Graf • \\ Dirk Boehmer • Volker Budach
}

Received: 27 June 2013/Accepted: 26 September 2013/Published online: 11 October 2013

(C) The International Gastric Cancer Association and The Japanese Gastric Cancer Association 2013

\begin{abstract}
Background The aim of this study was to determine the medical and technical feasibility of intensity-modulated radiotherapy (IMRT) in high-risk nonmetastatic gastric cancer stage II and III after primary gastrectomy and D2 lymphadenectomy.

Methods and materials A prospective nonrandomized phase II trial was performed on 25 consecutive patients with gastric cancer with high risk (T3-4, N1-3, G2-3, R0-1). The dose delivered was 45 Gy (1.80 Gy per fraction) in IMRT technique. Concurrent 5-fluorouracil-based chemotherapy at $225 \mathrm{mg} / \mathrm{m}^{2}$ was administered as a continuous intravenous infusion. Primary endpoints were acute gastrointestinal toxicity (CTC 4.0) and technical feasibility of IMRT in regard to dose planning and radiation delivery. Results Early acute events were defined as clinical and chemical adverse effects of IMRT and concurrent chemotherapy during treatment. By definition, 90 days after the end of IMRT has been evaluated as acute-phase toxicity. No patient had grade 4 or higher acute adverse events. Clinical grade 3 toxicity occurred in two patients $(8 \%)$ with diarrhea and in one case $(4 \%)$ with nausea. Hematological changes with grade 3 occurred in three cases $(12 \%)$ with hemoglobin decrease, in five cases $(25 \%)$ as leukopenia, and in one case (4\%) with thrombocytopenia. The mean dose for liver was $16 \mathrm{~Gy}$ and the percentage volume exceeding $30 \mathrm{~Gy}$ (V30) was $21 \%$. Mean dose for right and left kidney was 9 and $13 \mathrm{~Gy}$, respectively, and
\end{abstract}

H. Badakhshi $(\bowtie) \cdot$ A. Gruen · R. Graf · D. Boehmer .

V. Budach

Department for Radiation Oncology, Charité School of Medicine and University Hospital Berlin, Augustenburger Platz 1,

13353 Berlin, Germany

e-mail: Harun.Badakhshi@charite.de
V20 was $9 \%$ and $13 \%$, respectively. Heart received a median dose of $15 \mathrm{~Gy}$ and V40 was $17 \%$. The mean dose to the bowel was $11 \mathrm{~Gy}$ and V40 was $6 \%$. Spinal cord had at maximum $33 \mathrm{~Gy}$ in median. Specifics of dose distribution, including the coverage, for the target region were as follows: minimum was $33 \mathrm{~Gy}$, maximum $48.6 \mathrm{~Gy}$, and mean dose $44.6 \mathrm{~Gy}$. The prescribed dose (45 Gy) covered $99 \%$ and $95 \%$ of planning target volume (OTV) in $66 \%$ and $92 \%$ of cases, respectively. Median PTV was $15.77 \mathrm{ml}$ (range, 805-3,604 ml).

Conclusions The data support the practical feasibility of IMRT in adjuvant treatment in high-risk gastric cancer in the postoperative setting as a proof of principle. Acute toxicity has been tolerable.

Keywords Gastric cancer · Intensity-modulated radiotherapy $\cdot$ IMRT $\cdot$ Image-guided radiotherapy

\section{Introduction}

Surgery has been the treatment of choice for locally advanced gastric. However, even after complete resection and adequate D2 lymphadenectomy, high rates of locoregional recurrence continue to be reported [1-6]. Several approaches have been tried to improve the clinical outcome of resectable gastric cancer, including adjuvant chemotherapy (CT), concurrent radiochemotherapy (cRCT), and perioperative chemotherapy. Conventional radiotherapy ( ${ }_{\mathrm{con}} \mathrm{RT}$ ) combined with chemotherapy has been shown to decrease locoregional failure and improve overall survival in some retrospective studies on unresectable or residual disease after surgery. The landmark Intergroup 0116 trial [7] demonstrated an increase in the median overall survival from 27 to 36 months when postoperative conventional RT 
chemoradiation was used in addition to surgery. However, the trial was and still is criticized because of the low number of D2 lymphadenectomies and the high levels of toxicity. con RT in this trial was delivered mostly as twodimensional (2D) RT, although three-dimensional (3D) RT with large fields was used in some cases, and this was configured and verified by means of fluoroscopy and simple dose distribution models. This regimen led to significant acute and late abdominal toxicity, and $17 \%$ of the patients assigned to ${ }_{\text {con }} \mathrm{RCT}$ stopped treatment as a result. In addition, conRT may affect normal structures including the liver, kidneys, lungs, and heart, resulting in late morbidity. Postoperative target volumes may include the stomach bed (including surgical clips), a portion of the left hemi-diaphragm, and draining lymphatics, thereby putting all these structures at risk. The standard target dose of 45 Gy far exceeds the tolerance of several surrounding, critical normal tissues (most notably the kidneys and liver). As a result, con RT volumes are often significantly influenced by the need to avoid potential kidney and liver damage, but the resulting underdosing of some parts of the target can compromise local control and survival [8].

Intensity-modulated radiation therapy (IMRT) allows for more conformal dose delivery and selective sparing of critical structures such as the kidneys and liver, and may therefore allow for more complete target coverage to full dose; this in turn may substantially reduce acute toxicity and late sequelae. Theoretically, the improved target coverage and accuracy of IMRT could improve locoregional control and reduce toxicity.

The key to successful implementation of IMRT is to minimize the dose to the surrounding structures to prevent morbidity and compounded toxicity from chemotherapy while maintaining adequate coverage of the target by similar or even higher doses. This prospective feasibility study examined whether postoperative IMRT could be considered an effective and safe therapeutic option in clinical management of locally advanced gastric cancer.

\section{Materials and methods}

Between November 2008 and April 2011, 25 patients with newly diagnosed locally advanced gastric adenocarcinoma without distant metastases (T2-4 and N2-3, M0) were treated with postoperative IMRT and concurrent chemotherapy, all of whom were eligible for this study. All cases were discussed in a multidisciplinary team after surgery and selected by the team because of the risk factors related to T2-4 and N2-3. Patient and tumor characteristics are summarized in Table 1. The median age at the time of diagnosis was 60 years. Pretreatment evaluation included esophagogastroduodenoscopy, endoscopic ultrasonography, and
Table 1 Clinical and treatment characteristics

\begin{tabular}{|c|c|}
\hline Parameter & Number of patients \\
\hline \multicolumn{2}{|l|}{ Age (years) } \\
\hline$>60$ & 18 \\
\hline$<60$ & 7 \\
\hline \multicolumn{2}{|l|}{ Gender } \\
\hline Female & 5 \\
\hline Male & 20 \\
\hline \multicolumn{2}{|l|}{ Tumor location } \\
\hline Antrum & 11 \\
\hline Body & 11 \\
\hline Pylorus & 3 \\
\hline \multicolumn{2}{|l|}{ Grade } \\
\hline G1-2 & 3 \\
\hline G3 & 22 \\
\hline \multicolumn{2}{|l|}{ Tumor size } \\
\hline$>5 \mathrm{~cm}$ & 18 \\
\hline$<5 \mathrm{~cm}$ & 7 \\
\hline \multicolumn{2}{|l|}{ T stage } \\
\hline 3 & 20 \\
\hline 4 & 5 \\
\hline \multicolumn{2}{|l|}{$\mathrm{N}$ status } \\
\hline 1 & 5 \\
\hline 2 & 14 \\
\hline 3 & 6 \\
\hline \multicolumn{2}{|l|}{ Margin } \\
\hline R0 & 22 \\
\hline $\mathrm{R} 1$ & 3 \\
\hline \multicolumn{2}{|c|}{ Extent of nodal dissection } \\
\hline D2 & 25 \\
\hline D1 & 0 \\
\hline \multicolumn{2}{|l|}{ Extent of surgery } \\
\hline Total gastrectomy & 21 \\
\hline Subtotal gastrectomy & 4 \\
\hline \multicolumn{2}{|c|}{$\begin{array}{l}\text { Intensity-modulated radiotherapy (IMRT) } \\
\text { dose }\end{array}$} \\
\hline 45 Gy & 25 \\
\hline Other & 0 \\
\hline \multicolumn{2}{|l|}{ Chemotherapy } \\
\hline $5-\mathrm{FU}$ & 25 \\
\hline Other & 0 \\
\hline
\end{tabular}

computed tomography (CT) in all patients, as well as staging laparoscopy in 8 cases. Tumor stages were determined according the seventh edition of the American Joint Committee on Cancer staging system for gastric cancer, even for those treated before the new edition was published.

All patients received induction chemotherapy consisting of concurrent 5 -fluorouracil (5-FU) at a dose of $225 \mathrm{mg} / \mathrm{m}^{2}$ continuously administered intravenously. The actual IMRT 
dose was 45 Gy in all cases. Patients had been instructed not to eat or drink for $3 \mathrm{~h}$ before each treatment to reduce variability resulting from stomach distension. IMRT started 6 weeks after surgery.

\section{Target delineation}

The magnitude of RT in general and of IMRT in this study is affected by the definition of the high-risk zone of the upper abdomen in the adjuvant setting. Postoperatively, there is no actual gross tumor volume (GTV), because the stomach has been removed. However, in all cases we attempted to define a "GTV" according to the preoperative endoscopic and CT findings.

In high-risk situations, the clinical treatment volume (CTV) had to be as accurate as possible. The CTV included the GTV with a 3-cm mucosal expansion and involved the node, celiac axis, splenic hilum, porta hepatis, and subpyloric/retropancreatic regions for tumors involving the antrum. In 14 cases (56\%), we performed additionally fluoroscopy of the upper abdomen with oral contrast agent, and in 18 cases (72\%), ultrasonography was performed to define the CTV more precisely. Positron emission tomography/CT was performed in addition to more conventional staging procedures in 12 cases $(48 \%)$ in which distant metastases were suspected.

The CTV was expanded circumferentially by $10 \mathrm{~mm}$ to form the planning target volume (PTV). Image guidance with daily kilovolt imaging was used for positioning according to the bony anatomy in all cases. Inverse treatment planning was performed using Eclipse and later ARIA oncology information systems (Varian Medical Systems, Palo Alto, CA, USA) and the direct machine parameter optimization algorithm. Generally, we used a median of five beams with an anterior or left anterior oblique beam angle configuration that would directly enter the stomach and exit posteriorly, thus sparing critical organs (Figs. 1 and 2). The V20 for each organ was the volume (percentage) receiving at least $20 \mathrm{~Gy}$; V30, that receiving at least $30 \mathrm{~Gy}$; and $\mathrm{V} 40$, that receiving at least 40 Gy. Normal tissue constraints included a V30 less than $30 \%$ and a V20 less than $50 \%$ for the liver, a V20 lower than $25 \%$ for each kidney, a V40 less than $25 \%$ and a V30 less than $35 \%$ for the heart, and a maximum spinal cord dose of $45 \mathrm{~Gy}$. The dose to the lungs and the left ventricle was reduced as much as possible.

Logistics and follow-up examination

The median time between surgery and IMRT was 5 weeks. During IMRT all patients were seen by a member of the study team (HB) at least once a week. Acute toxicity was

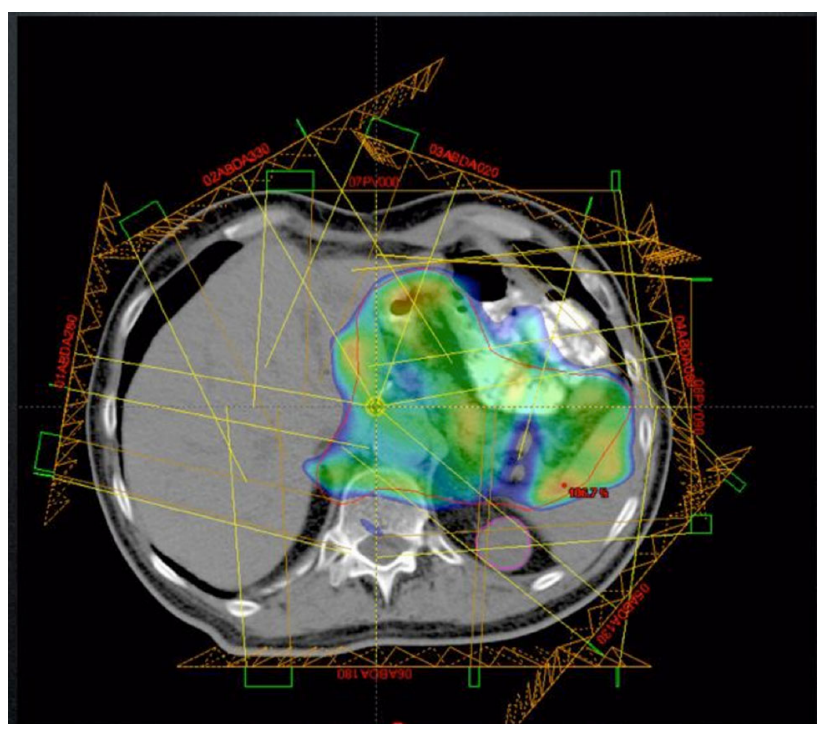

Fig. 1 Clinical target volume based on computed tomography (CT)

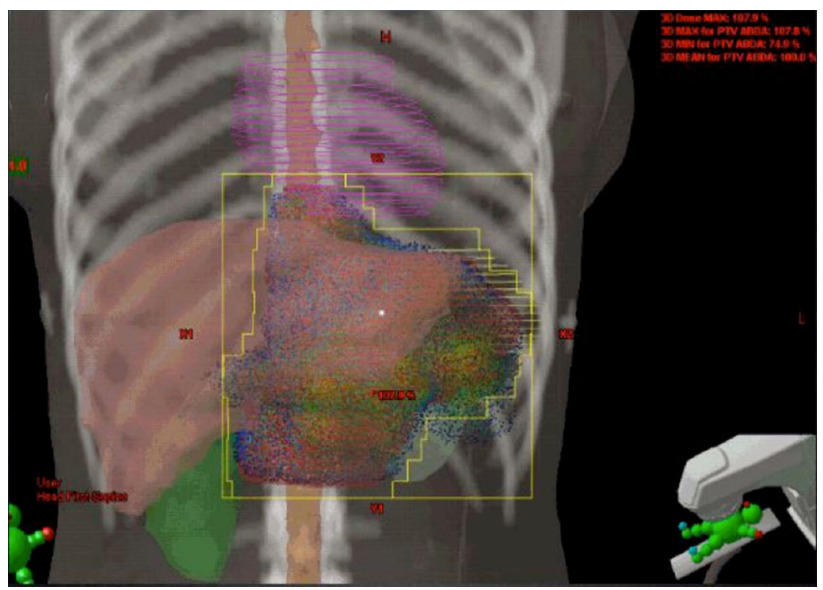

Fig. 2 Clinical target volume and field arrangement based on CT

graded according to the Common Terminology Criteria (CTC) for Adverse Events version 4.0.

Follow-up information was obtained from patient records and clinic notes.

The primary endpoint of this prospective feasibility study was acute toxicity and technical performance with regard to dose distribution to the PTV and organs at risk.

\section{Results}

Primary endpoint: acute toxicity

Early acute events were defined as clinical adverse effects of IMRT and concurrent chemotherapy during treatment, with acute-phase toxicity being defined as that occurring 
Table 2 Acute toxicity

\begin{tabular}{lrrrrr}
\hline & \multicolumn{3}{l}{$\begin{array}{l}\text { Grade (CTC 4.0), number of } \\
\text { patients }\end{array}$} & \multirow{2}{l}{ Total grade $>2$} \\
\cline { 2 - 5 } & 1 & 2 & 3 & 4 & \\
\hline Nausea & 12 & 12 & 1 & 0 & 1 \\
Vomiting & 10 & 10 & 0 & 0 & 0 \\
Diarrhea & 4 & 4 & 2 & 0 & 2 \\
Hb decrease & 8 & 10 & 3 & 0 & 3 \\
Leukopenia & 5 & 5 & 5 & 0 & 5 \\
Thromocytopenia & 4 & 3 & 1 & 0 & 1 \\
Pain & 3 & 4 & 0 & 0 & 0 \\
\hline
\end{tabular}

within 90 days of the completion of IMRT. No patient had a grade 4 or worse acute adverse event, but clinical grade 3 toxicity was experienced by two patients $(8 \%)$ with diarrhea, one (4\%) with nausea, three $(12 \%)$ with reduced hemoglobin levels, five (20\%) with leukopenia, and one (4\%) with thrombocytopenia (Table 2).

\section{Primary endpoint: dosimetric characteristics of IMRT}

The mean dose for liver was $16 \mathrm{~Gy}$ and the percentage volume exceeding 30 Gy (V30) was $21 \%$. Mean dose for right and left kidney was 9 and $13 \mathrm{~Gy}$ and V20 was $9 \%$ and $13 \%$, respectively. Heart received a median dose of $15 \mathrm{~Gy}$ and V40 was $17 \%$. The mean dose to the bowel was 11 Gy and V40 was $6 \%$. The spinal cord had at maximum 33 Gy in median.

Specifics of dose distribution, including the coverage, for the target region was as it follows: minimum was $33 \mathrm{~Gy}$, maximum $48.6 \mathrm{~Gy}$, and mean dose $44.6 \mathrm{~Gy}$.

The prescribed dose (45 Gy) covered $99 \%$ and $95 \%$ of planning target volume (OTV) in $66 \%$ and $92 \%$ of cases, respectively. Median PTV was $15.77 \mathrm{ml}$ (range, $805-3,604 \mathrm{ml})$.

Secondary endpoint: local control

The median follow-up period was 16 months (range, 10-35 months). A year after completing IMRT, three patients $(12 \%)$ experienced local failure and another two experienced regional relapse $(8 \%)$. Distant metastases developed in six patients $(24 \%)$, five of whom died $(20 \%)$.

\section{Discussion}

This study was designed to assess the acute toxicity resulting from IMRT by using a validated standardized score (CTC 4.0) and by evaluating the dosimetric characteristics for organs at risk and the upper abdominal region of the tumor. We also examined the short-term clinical outcome, albeit as a secondary endpoint. There were no cases of grade 4 or worse adverse effects during combined IMRT and 5-FU-based chemotherapy, although there was a high incidence $(12 \%)$ of grade 3 clinical toxicity, most cases of which involved hematological changes including leukocytopenia $(25 \%)$ and reduced hemoglobin levels (12\%). All patients were treated in the clinical ward of the radiation oncology department, with strict clinical control and observation, and there were no interruptions to therapy, except in three cases with an IMRT delay of 2-3 days. Thus, therapy was more continuous than is usually achieved with con RT [7-10].

Although IMRT does not provide a significant reduction in acute toxicity compared to con RT, it does spare the kidneys and liver, as demonstrated in this study as well as previous studies [11-13]. The sparing of critical organs is clinically very important because concurrent chemotherapy also affects the kidneys, liver, and parts of the spinal cord and because it reduces potential long-term toxicity, especially for patients undergoing major abdominal surgery and for those who may subsequently need further chemotherapy.

Our findings are generally consistent with those of other studies on IMRT for gastric cancer. A previous comparison of patients treated with ${ }_{\text {con }} \mathrm{RT}$ or IMRT indicated that IMRT reduces the dose to the liver but does not reduce acute gastrointestinal toxicity [11]. This finding was supported by another study in which IMRT was also shown to provide better protection of the liver and kidneys compared with 3D CRT [12], although this conflicts with a study that showed only marginal sparing of the kidneys [13].

Preoperative IMRT was previously shown to give rise to acute adverse effects of grade 3 acute toxicity in 14 patients (56\%), including dehydration in 10 cases, nausea in 8 cases, and anorexia in 5 cases, although there were no cases of grade 4 toxicity. The same study showed that the median target coverage was 0.97 (range, 0.92-1.01), with a median V30 for the liver of $26 \%$; a median V20 for the right and left kidneys of 14 and $24 \%$, respectively; and a median V40 (percentage of volume receiving at least $40 \mathrm{~Gy}$ ) for the heart of $18 \%$ [14]. In our study, we found that the doses to the left kidney and heart were lower than these previously reported values.

A number of other studies have also reported that IMRT is advantageous compared to con RT. Jansen et al. [15] showed that if $20 \%$ of one kidney receives a dose of more than $20 \mathrm{~Gy}, 11 \%$ and $52 \%$ of these patients will suffer from renal insufficiency within 6 months and 1 year of RT, respectively. Milano et al. treated seven gastric cancer patients with IMRT and compared the IMRT plan to that for ${ }_{\text {con }} \mathrm{RT}$. The former was found to have an advantageous 
dose distribution in the target area and to provide better protection of high-risk organs [8].

In conclusion, our findings suggest that IMRT is a good option and a feasible method. The use of advanced IMRT techniques makes the side effects tolerable and manageable by protecting at-risk organs while providing good coverage of the target volume.

IMRT, combined with chemotherapy, may be considered a safe therapeutic option in addition to surgery in locally advanced gastric cancer. We encourage more prospective evidence generation in terms of testing effectiveness.

Acknowledgments We, all authors, confirm that there has been no funding to support the research in regard to this paper.

Conflict of interest All authors confirm that there is no conflict of interest to any financial and personal relationships with other people or organization.

\section{References}

1. Gunderson LL, Sosin H. Adenocarcinoma of the stomach: areas of failure in a re-operation series (second or symptomatic look) clinicopathologic correlation and implications for adjuvant therapy. Int J Radiat Oncol Biol Phys. 1982;8:1-11.

2. Landry J, Tepper JE, Wood WC, Moulton EO, Koerner F, Sullinger J. Patterns of failure following curative resection of gastric carcinoma. Int J Radiat Oncol Biol Phys. 1990;19:1357-62.

3. Papachristou DN, Fortner JG. Local recurrence of gastric adenocarcinomas after gastrectomy. J Surg Oncol. 1981;18:47-53.

4. Maehara Y, Hasuda S, Koga T, Tokunaga E, Kakeji Y, Sugimachi K. Postoperative outcome and sites of recurrence in patients following curative resection of gastric cancer. Br J Surg. 2000;87:353-7.

5. Yoo CH, Noh SH, Shin DW, Choi SH, Min JS. Recurrence following curative resection for gastric carcinoma. $\mathrm{Br} \mathrm{J}$ Surg. 2000;87:236-42.
6. Lim DH, Kim DY, Kang MK, Kim YI, Kang WK, Park CK, et al. Patterns of failure in gastric carcinoma after D2 gastrectomy and chemoradiotherapy: a radiation oncologist's view. Br J Cancer. 2004;91:11-7.

7. Macdonald JS, Smalley SR, Benedetti J, Hundahl SA, Estes NC, Stemmermann GN, et al. Chemoradiotherapy after surgery compared with surgery alone for adenocarcinoma of the stomach or gastroesophageal junction. N Engl J Med. 2001;345:725-30.

8. Milano MT, Garofalo MC, Chmura SJ, Farrey K, Rash C, Heimann $\mathrm{R}$, Jani $\mathrm{AB}$. Intensity-modulated radiation therapy in the treatment of gastric cancer: early clinical outcome and dosimetric comparison with conventional techniques. $\mathrm{Br} \mathrm{J}$ Radiol. 2006;79(942):497-503.

9. Kassam Z, Lockwood G, O'Brien C, et al. Conformal radiotherapy in the adjuvant treatment of gastric cancer: review of 82 cases. Int J Radiat Oncol Biol Phys. 2006;65:713-9.

10. Chang AT, Ng WT, Law AL, et al. Adjuvant chemoradiation for resected gastric cancer: a 10-year experience. Gastric Cancer. 2011;14:63-71.

11. Minn AY, Hsu A, La T, et al. Comparison of intensity-modulated radiotherapy and 3-dimensional conformal radiotherapy as adjuvant therapy for gastric cancer. Cancer (Phila). 2010;116: 3943-52.

12. Dahele M, Skinner M, Schultz B, et al. Adjuvant radiotherapy for gastric cancer: a dosimetric comparison of 3-dimensional conformal radiotherapy, tomotherapy and conventional intensity modulated radiotherapy treatment plans. Med Dosim. 2010;35: 115-21.

13. Alani S, Soyfer V, Strauss N, et al. Limited advantages of intensity-modulated radiotherapy over 3D conformal radiation therapy in the adjuvant management of gastric cancer. Int $\mathbf{J}$ Radiat Oncol Biol Phys. 2009;74:562-6.

14. Chakravarty T, Crane CH, Ajani JA, Mansfield PF, Briere TM, Beddar AS, Mok H, Reed VK, Krishnan S, Delclos ME, Das P. Intensity-modulated radiation therapy with concurrent chemotherapy as preoperative treatment for localized gastric adenocarcinoma. Int J Radiat Oncol Biol Phys. 2012;83(2):581-6.

15. Jansen EP, Saunders MP, Boot H, Oppedijk V, Dubbelman R, Porritt B, Cats A, Stroom J, Valdes OR, Bartelink H, Verheij M. Prospective study on late renal toxicity following postoperative chemoradiotherapy in gastric cancer. Int $\mathrm{J}$ Radiat Oncol Biol Phys. 2007;67:781-5. 